\title{
Gastric coccidioidomycosis presenting as gastric outlet obstruction
}

Taher Jamali ${ }^{1}$, Kati Choi ${ }^{2}$, Syed Tasleem ${ }^{2}$, Neda Zarrin-Khameh ${ }^{3}$, and Robert J. Sealock ${ }^{2}$

${ }^{1}$ Department of Internal Medicine, ${ }^{2}$ Division of Gastroenterology and Hepatology, Department of Internal Medicine, ${ }^{3}$ Department of Pathology \& Immunology, Baylor College of Medicine, Houston, TX, USA

Received : December 27, 2020

Revised : March 1, 2021

Accepted: March 1, 2021

\section{Correspondence to}

Taher Jamali, M.D.

Tel: +1-832-566-2661

Fax: +1-281-357-1057

E-mail: taherjamali110@gmail.com https://orcid.org/0000-0001-

6479-2762

\begin{abstract}
A previously healthy, 36-year-old Latino human immunodeficiency virus-negative man from the Southern United States with no recent travel history presented with one month of abdominal pain, nausea, vomiting, post-prandial emesis, and unintentional weight loss. He denied smoking, use of non-steroidal anti-inflammatory drugs, and history of Helicobacter pylori infection. Abdominal imaging showed a $2.2-\mathrm{cm}$ gastric antral mass with evidence of gastric outlet obstruction (Fig. 1A). Upper endoscopy identified an ulcerated pyloric channel mass with raised edges causing gastric outlet obstruction (Fig. 1B). Biopsies from the ulcerated mass
\end{abstract}

demonstrated necroinflammatory debris, round structures with well-defined boundaries, and underlying stroma with increased eosinophils. Periodic acid-Schiff (PAS) and Grocott's methenamine silver stains were negative, and there were no signs of underlying malignancy. Repeat upper endoscopy with biopsies revealed similar findings. Despite conservative management with nasogastric tube decompression and intravenous proton pump inhibitor therapy, the patient's symptoms persisted, and he underwent distal gastrectomy with loop gastrojejunostomy. By the third day after the surgery, the patient was tolerating regular diet. Fi-
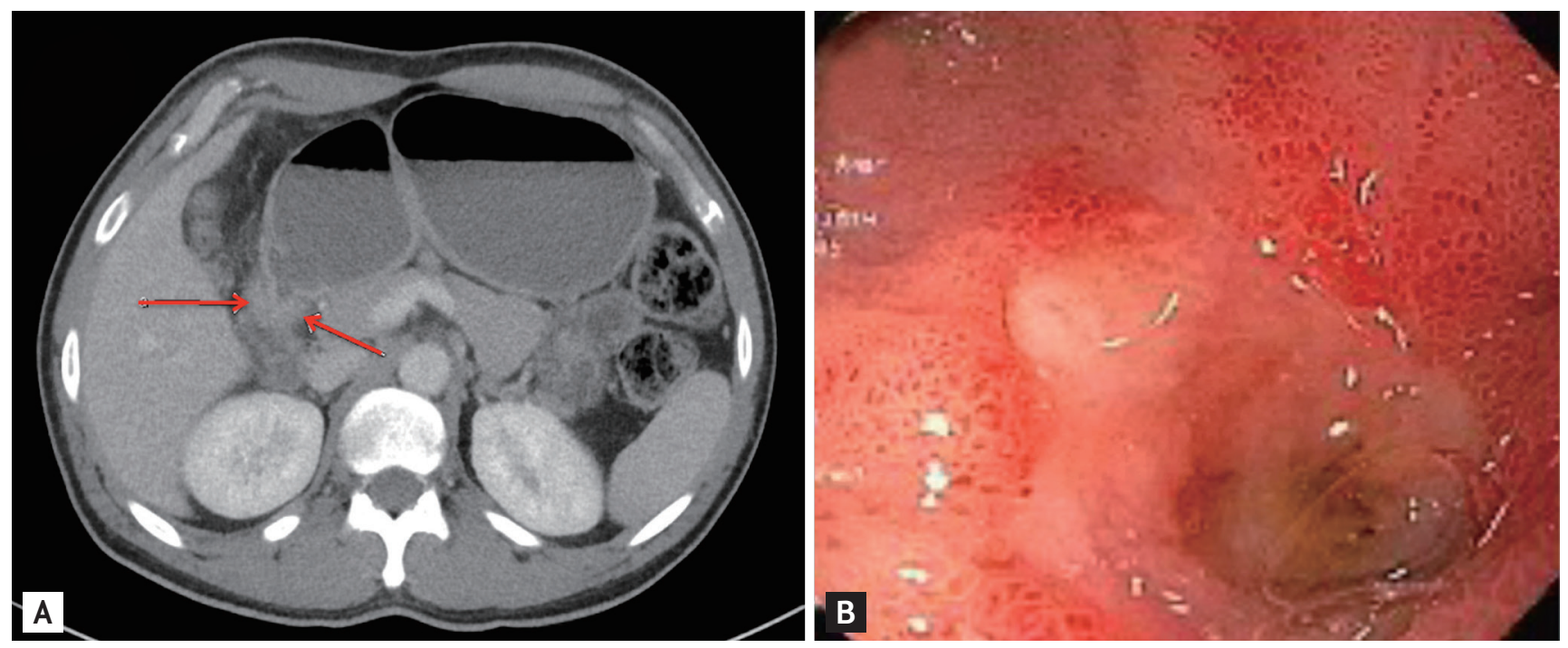

Figure 1. (A) Computed tomography imaging showing a 2.2-centimeter gastric antral mass with evidence of gastric outlet obstruction (arrows), (B) esophagogastroduodenoscopic findings of an ulcerated pyloric channel mass with raised edges. 


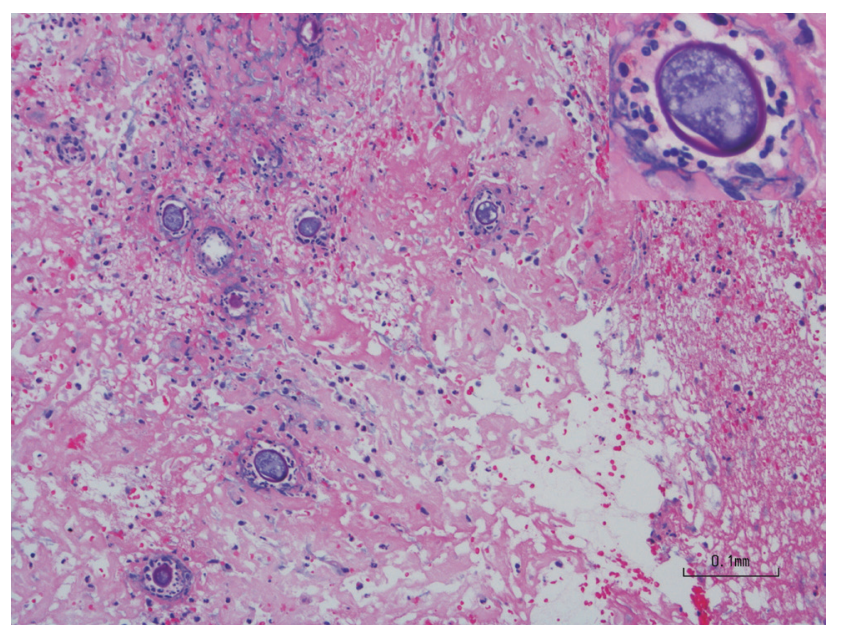

Figure 2. Periodic acid-Schiff stain from final surgical pathology highlighting large coccidioidomycosis spherules (up to 80 microns) with many endospores and evidence of chronic active gastritis.

nal surgical pathology revealed coccidioidomycosis-associated chronic active gastritis and gastric ulcers. PAS stains showed large coccidioidomycosis spherules (up to 80 microns) with many endospores (Fig. 2). The patient's symptoms had significantly improved post-operatively, and after the diagnosis of coccidioidomycosis was established, treatment was initiated with fluconazole $400 \mathrm{mg}$ daily for 2 months. Outpatient follow-up was requested in the infectious diseases clinic. The diverse clinical manifestations of coccidioidomycosis make diagnosis particularly challenging. Gastrointestinal coccidioidomycosis is especially rare and there are scarce reports of cases presenting as small bowel obstruction or as gastric ulcers. In order to increase clinician awareness, we highlight a very distinctive gastrointestinal manifestation of coccidioidomycosis presenting as gastric outlet obstruction.

\section{Conflict of interest}

No potential conflict of interest relevant to this article was reported. 